\title{
Factores psicosociales del personal de una organización educativa y su relación con desgaste psíquico
}

\author{
Psychosocial Factors in Workers of an Educational Organization \\ and their Relationship with Psychic Exhaustion
}

\author{
Ana Anaya-Velasco 1
(iD) https://orcid.org/0000-0003-1137-9645 \\ Estibalis Anaís Luna Chávez² \\ https://orcid.org/0000-0003-4296-6295 \\ Claudia Saldaña Orozco ${ }^{3}$
https://orcid.org/0000-0003-3268-7209
M. Ángeles Carrión-García ${ }^{4}$
https://orcid.org/0000-0003-4479-3973

\footnotetext{
1,3 Departamento de Promoción, Preservación y Desarrollo de la Salud, Universidad de Guadalajara, México ²Posgrado en Psicología con Orientación en Calidad de Vida y Salud, Universidad de Guadalajara, México ${ }^{4}$ Psicología y Educación, Universitat Oberta de Catalunya, España
}

Resumen. Objetivo. Evaluar los factores psicosociales percibidos por los trabajadores en una organización pública de educación superior y su relación con el desgaste psíquico. Método. La investigación fue transversal, participaron 292 trabajadores y se utilizó una batería que evalúa las dimensiones: Contexto de Trabajo, Contenido de Trabajo y Factores Individuales, como variables independientes y Desgaste Psíquico como variable dependiente. Resultados. La percepción de los participantes resultó favorable en más del $80 \%$. Las dimensiones psicosociales indicaron una correlación media, positiva y estadísticamente significativa con el Desgaste Psíquico, consistente con los análisis Odds Ratio. Factores Individuales fue la dimensión de mayor influencia acorde con el modelo de regresión lineal múltiple, seguida de Contenido de Trabajo.

Palabras clave. Factores psicosociales, seguridad y salud en el trabajo, organización educativa.

Abstract. Objective. To evaluate the psychosocial factors perceived by workers in a higher education public organization and their relationship with the psychic exhaustion. Method. Research was cross-sectional, 292 workers participated and it was used a test battery to evaluate the dimensions: Work Context, Work Content and Individual Factors, as independent variables and Psychic Exhaustion as a dependent variable. Results. Perception of the participants was favorable by more than $80 \%$. Psychosocial dimensions indicated a medium, positive and statistically significant correlation with the Psychic Exhaustion, consistent with Odds Ratio analysis. Individual Factors was the dimension with the greatest influence according to the multiple linear regression model, followed by Work Content.

Keywords. Psychosocial Factors, Occupational Safety and Health, Educational Organization.

${ }^{1}$ Ana Anaya-Velasco. Departamento de Promoción, Preservación y Desarrollo de la Salud, Universidad de Guadalajara, México. Dirección postal: Centro Universitario del Sur. Av. Enrique Arreola Silva No. 883, Colonia Centro, C.P. 49000, Ciudad Guzmán, Jalisco, México. E-mail: anayav@cusur.udg.mx

Estibalis Anaís Luna Chávez. Posgrado en Psicología con Orientación en Calidad de Vida y Salud, Universidad de Guadalajara, México. E-mail: eanaisluna@hotmail.com

${ }^{3}$ Claudia Saldaña Orozco. Posgrado en Psicología con Orientación en Calidad de Vida y Salud, Universidad de Guadalajara, México. E-mail: claudias@cusur.udg.mx

${ }^{4}$ M. Ángeles Carrión-García. Psicología y Educación, Universitat Oberta de Catalunya, España. E-mail: mcarrionga@uoc.edu

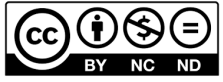

Esta obra está bajo una licencia de Creative Commons Reconocimiento-NoComercial-SinObraDerivada 4.0 Internacional. 


\section{Introducción}

Ante la situación de los riesgos de trabajo que dañan al personal, como lo señalan la Organización Internacional del Trabajo (OIT, 2021) y la Organización Mundial de la Salud (OMS, 2006), se plantean desafíos de calidad, productividad y sustentabilidad, asociados a las condiciones del ambiente físico, psicosocial y de la organización del trabajo, así como del entorno natural y social. Es necesario abordar el estudio y aplicación de procesos de mejora basados en esfuerzos unidos desde una gestión integral, como lo han propuesto algunos organismos internacionales, entre ellos la Organización Internacional de Normalización (ISO, 2010) y autores como Carrión et al. (2008) y Salanova et al. (2013, 2021).

No obstante, aun cuando pueda parecer complejo, el origen de los riesgos está en el lugar de trabajo donde el personal realiza las actividades laborales propias del puesto, y es ahí mismo donde los riesgos pueden disminuirse (Anaya, 2017). Como lo señala Chiavenato (2011) no es ético que las personas malogren su salud intentando ganarse la vida.

Un factor de riesgo en el ámbito de la salud es cualquier rasgo, característica o exposición de un individuo que aumente su probabilidad de sufrir una enfermedad o lesión (OMS, 2017). En el ámbito laboral, Gutiérrez (2011) lo define como las condiciones del ambiente, instrumentos, materiales, la tarea o la organización del trabajo que encierra un daño potencial a la salud de los trabajadores o un efecto negativo en la empresa, estos incluyen a los factores químicos, físicos, biológicos, ergonómicos, mecánicos, eléctricos y psicosociales. En este sentido, Andersen et al. (2020) identificaron que el esfuerzo físico y las demandas emocionales se asociaron con el riesgo de pensión por discapacidad.

Los factores psicosociales empiezan a causar mayor interés por su influencia en la salud de los trabajadores, en su desempeño y en su calidad de vida laboral (Patlán, 2016), por lo cual son contemplados en las legislaciones de muchos países e impulsa a las organizaciones a prevenir riesgos para la salud física y psicológica de sus empleados.

Desde décadas atrás, se ha tratado de explicar la relación de las condiciones del trabajo con la salud de los empleados, así se identifican tres enfoques que se superponen y que toman como desencadenante de las alteraciones de la salud a la presencia de estrés laboral. El primero, llamado enfoque técnico concibe al estrés derivado del trabajo como una característica nociva del entorno, en términos de carga, exigencias o elementos amenazantes. El enfoque fisiológico postula que el estrés es un síndrome fisiológico generalizado e inespecífico cuyo origen es la activación de sistemas neuroendocrinos en respuesta a estímulos dañinos. Por último, el enfoque psicológico establece que el estrés resulta de la interacción entre la persona y el entorno, mediados por los procesos cognitivos y las reacciones emocionales en las que se dan estas interacciones (Karasek, 1979; Lazarus, 1974; Selye, 1946).

El enfoque psicológico es al que se alinea la definición de los factores psicosociales emitido por la OIT (Cox et al., 2005) y se plasma en las legislaciones nacionales como la de México (Ley Federal del Trabajo, 2012; Reglamento Federal de Seguridad y Salud en el Trabajo, 2014; Norma Oficial Mexicana NOM-035-STPS-2018, 2018).

Se identifican tres términos en la literatura de salud ocupacional: factores psicosociales, factores de riesgo psicosocial y riesgos psicosociales; estos conceptos muestran la complejidad del tema (Moreno \& Báez, 2010) y tienen variaciones que deben considerarse.

De acuerdo con el informe del Comité Mixto de la OIT y la OMS de Medicina del Trabajo, Factores psicosociales se entienden como "las interacciones entre el contenido, la 
organización, gestión del trabajo y condiciones ambientales, por un lado, y las capacidades y necesidades de los trabajadores, por el otro" (1984, p. 3), además, precisa que "estas interacciones podrían ejercer una influencia nociva para la salud de los trabajadores a través de sus percepciones y experiencia" (Comité Mixto de la OIT/OMS de Medicina del Trabajo, 1984, p. 12).

Factores de riesgo psicosocial son los factores psicosociales que ejercen una influencia nociva y pueden afectar tanto al trabajador como a la organización (Cacivio, 2017; Ramalho \& Costa, 2017). La exposición a ellos es una fuente generadora de estrés y posibilita la aparición de desgaste psíquico determinado por la sintomatología relacionada de carácter físico, cognitivo-emocional y conductual (Carrión, 2014; Xiao et al., 2020); se derivan de las deficiencias en el diseño, la organización, la gestión, la comunicación y el contexto social del trabajo (Agencia Europea para la Seguridad y la Salud en el Trabajo, 2016; Hammer et al., 2015).

Los factores de riesgo psicosocial en México han sido recientemente incorporados a la legislación. El Reglamento Federal de Seguridad y Salud en el Trabajo (2014) estableció la necesidad de regular el diagnóstico, detección, tratamiento y reducción de los factores de riesgo psicosocial para lograr la salud en el trabajo y la inclusión de sus efectos en la tabla de enfermedades de trabajo (arts. $2^{\circ}$ y 73 ). Posteriormente, la Norma Oficial Mexicana NOM-035-STPS-2018 (2018) señala entre los factores de riesgo psicosocial: las condiciones peligrosas, las cargas excesivas, la baja posibilidad de influir, las jornadas que sobrepasan la duración prevista en la Ley Federal del Trabajo (2012), la interferencia trabajo-familia, el liderazgo y las relaciones negativas en el trabajo.

Riesgos psicosociales del trabajo es el tercer concepto identificado en la literatura, difiere de factores psicosociales y factores de riesgo psicosocial, porque se trata de los daños a la salud de los trabajadores, tales como la ansiedad, la depresión, los trastornos del sueño, el estrés grave (Norma Oficial Mexicana NOM-035-STPS-2018, 2018), el desgaste psíquico (Carrión, 2014), problemas cardiovasculares, el dolor de espalda y otros trastornos músculo esqueléticos, trastornos médicos de tipo respiratorio, gastrointestinal entre otros, así como conductas sociales como el hábito de fumar, consumo de drogas, sedentarismo, falta de participación y el ausentismo laboral.

El Desgaste Psíquico es la respuesta subjetiva cognitivo-emocional, conductual y fisiológica, derivado del esfuerzo de atención, el esfuerzo de concentración o reflexión necesarios para recibir la información del proceso y elaborar las respuestas adecuadas y por la constancia con que debe ser sostenido este esfuerzo, en conjunto con la fatiga percibida, los síntomas subjetivos y alteraciones de la salud como consecuencias que se desprenden de una sobrecarga de las exigencias de la tarea, el número de informaciones que se precisan para realizar la tarea y el nivel de complejidad de las mismas; y finalmente, la percepción de la dificultad que para el trabajador tiene su trabajo (Martín et al., 1995).

Los estudios enfocados a la salud laboral, a los aspectos psicosociales y organizacionales han cobrado importancia y reconocimiento en los últimos años. Los investigadores se han dedicado a detectar, evaluar y determinar la repercusión nociva o favorable en la salud física, mental y social de los trabajadores (Moreno \& Báez, 2010; Tamayo et al., 2014). La etapa siguiente a la identificación y evaluación de los factores de riesgo psicosocial es el diseño e implementación de medidas de mejora o programas de intervención. Si bien, tanto la ergonomía como la seguridad e higiene pueden intervenir en los factores de riesgo laborales para aminorarlos, para los factores psicosociales las 
soluciones no resultan ser claras y representan un desafío para las organizaciones. Por lo que planificar una intervención desde un enfoque integrado en medicina, salud pública y psicología resulta conveniente para abordar problemas comunes de la salud en el trabajo (LaMontagne et al., 2014; Moreno \& Báez, 2010).

En el ámbito internacional se identificaron algunos estudios, en los cuales, a partir de una evaluación de factores psicosociales se plantearon medidas o programas para la mejora tanto para la salud del trabajador como para la organización (Tomascheck et al., 2017; Vestly et al., 2017).

Desde una perspectiva integral han surgido modelos que buscan beneficios compartidos tanto para el trabajador como para las organizaciones y el entorno, como el modelo AEPA (Carrión, 2008), la Guía de responsabilidad social de la International Standarization Organization (ISO, 2010), Entornos laborales saludables (OMS, 2010), el modelo Healthy and Resilient Organizations (Salanova et al., 2013) y el modelo SSeTGIS (Anaya, 2017).

Para estudiar los factores psicosociales e implementar medidas con visión integral, se han desarrollado instrumentos alrededor del mundo que buscan evaluar la percepción del trabajador respecto a factores de riesgo psicosocial, el CoPsoQ-istas21 del Instituto Sindical del Trabajo, Ambiente y Salud (ISTAS, 2016), su actualización, el COPSOQ III (Burr et al., 2019; Berthelsen et al., 2020), el F-PSICO (Instituto Nacional de Seguridad e Higiene en el Trabajo [INSHT], 2014) y la batería CTCPS-MAC (Carrión, 2014).

La batería CTCPS-MAC evalúa condiciones de trabajo de carácter psicosocial en cuatro dimensiones o factores psicosociales globales que se integran por factores psicosociales específicos. Estudios realizados con la batería CTCPS-MAC (Carrión et al., 2014; Saldaña et al., 2014) muestran la percepción desfavorable en Contexto de Trabajo y en Contenido de Trabajo en las que los participantes tienen mayor probabilidad de sufrir Desgaste Psíquico, en el que identificaron aspectos favorables de las condiciones de trabajo y su incidencia en la organización y el trabajador. Además, se registraron casos nocivos en los factores Papel o Rol en la Organización y Respuesta Fisiológica, por lo que para ambos estudios se propuso un proceso de intervención.

El presente estudio se realizó con el objetivo de evaluar los factores psicosociales percibidos por los trabajadores en una organización pública de educación superior y su relación con el desgaste psíquico.

La institución se ubica en una ciudad del sur de Jalisco, México, atiende a 7600 estudiantes, ha tenido un crecimiento acelerado en los últimos años con un incremento en la carga laboral del personal y en la complejidad de las actividades para acceder o conservar estímulos al desempeño, con pocas posibilidades de promoción y sin plazos definidos. En el contrato colectivo de trabajo se obliga a brindar espacios seguros y saludables para el desempeño de sus actividades. La responsabilidad social universitaria y calidad de vida es un eje de su política como universidad sostenible.

La investigación da respuesta a las siguientes preguntas: ¿cuáles factores psicosociales globales y específicos fueron percibidos por el personal de la institución como favorables y cuáles como desfavorables?, ¿se presenta una correlación estadísticamente significativa en la percepción de las dimensiones Contexto de Trabajo, Contenido de Trabajo y Factores Individuales con el Desgaste Psíquico de los participantes?, ¿cuál de las dimensiones psicosociales influye más en el Desgaste Psíquico de acuerdo con el modelo de regresión lineal múltiple? 


\section{Método}

El estudio fue cuantitativo, no experimental, transversal, de tipo correlacional.

\section{Participantes}

La muestra fue no probabilística e incluyó a 292 trabajadores de una plantilla que rebasa los 700, de estos, un tercio tenían contrato permanente de jornada completa, los restantes eran administrativos y operativos por contrato temporal así como profesores por horas. Los participantes aceptaron contribuir de manera voluntaria con el estudio, respondiendo a la convocatoria que para tal efecto se hizo por diferentes medios y por invitación directa a quienes estuvieron presentes en su lugar de trabajo en la primera o una segunda visita con base en el listado de personal.

De la muestra $46 \%$ fueron mujeres y $54 \%$ hombres, el estado civil reportado por la mayoría fue casado con 58\% y la formación académica fue de maestría con el 34\%, licenciatura $29 \%$, doctorado $13 \%$, mientras que $14 \%$ tenían bachillerato y $10 \%$ menor escolaridad. La media y desviación estándar en años fueron: para edad de 42.59 y 10.845 , para antigüedad de 12.71 y 8.920 y en el puesto actual de 8.22 y 7.778 . El puesto de profesor lo ocupan $63.44 \%$ (asignatura por horas $18.62 \%$, investigador $14.83 \%$, docente $12.76 \%$, con cargo administrativo o técnico $10.34 \%$ y con cargo directivo $6.89 \%$ ) el $36.55 \%$ son operativos y auxiliares administrativos.

\section{Instrumento}

Se utilizó la batería para el estudio de las Condiciones de Trabajo de Carácter Psicosocial (CTCPS-MAC), desarrollada por Carrión (2014), compuesta por 75 ítems con respuesta en escala tipo Likert cuyas opciones de respuesta van de "completamente de acuerdo" a "completamente en desacuerdo". Los ítems se agrupan en cuatro dimensiones o factores psicosociales globales que se evalúan por separado y 14 factores psicosociales específicos que evalúan la percepción que de ellos tiene la persona a quien se aplica, en niveles de "muy bueno", "bueno", "normal", "regular" y "nocivo".

El instrumento reporta una confiabilidad óptima con un coeficiente alfa de Cronbach de .92, el cual fue validado y adaptado para población iberoamericana en un estudio anterior, en el que participaron 1266 trabajadores de los cuales el 28.9\% pertenecía a la población mexicana Carrión (2014). Se utilizó un análisis de componentes principales, con el método de rotación, normalización Varimax con Kaiser, se tomaron como significativas las cargas factoriales superiores a .5 en valor absoluto. Los resultados mostraron que los ítems se agrupan en los 14 factores que integran el instrumento y la varianza total explicada fue de 56.083\%. El valor de Kaiser-Meyer-Olkin (KMO) fue .927, esta medida es un indicador de fuerza entre las variables. Un valor cercano a uno indica que el análisis factorial es posible, asimismo la prueba de esfericidad de Barlett tuvo una significación de .001 lo que sugiere que la matriz es adecuada para ejecutar este mismo análisis.

Se categoriza para algunos análisis como favorable y probablemente protector, el factor psicosocial percibido en niveles de muy bueno y bueno; mientras que, como desfavorable y probable factor de riesgo psicosocial, el percibido en niveles de normal, regular y nocivo, ya que indican que se hacen necesarias medidas de mejora, reparadoras e inmediatas para evitar el daño.

Las tres primeras dimensiones son variables independientes: Contexto de Trabajo, Contenido de Trabajo y Factores Individuales, mientras la cuarta dimensión que es 
Tabla 1

Dimensiones y factores de la batería Condiciones de Trabajo de Carácter Psicosocial

\begin{tabular}{ll}
\hline \multicolumn{1}{c}{ Dimensión } & \multicolumn{1}{c}{ Factores psicosociales } \\
\hline D1. Contexto de Trabajo & D1-F1. Cultura de la Organización y Gestión \\
& D1-F2. Papel o Rol en la Organización \\
& D1-F3. Interrelación Trabajo con Problemas Familiares o Sociales \\
D2. Contenido de Trabajo & D1-F4. Relaciones Interpersonales \\
& D2-F1. Carga y Ritmo de Trabajo \\
& D2-F2. Ambientes Laborales \\
& D2-F3. Equipos y Agentes Físicos \\
& D2-F4. Concepción de las Tareas del Puesto de Trabajo \\
& D3-F1. Amortiguadores del Riesgo Psíquico \\
& D3-F2. Características de la Empresa \\
D3. Factores Individuales & D3-F1. Cíntomas subjetivos y alteraciones de la salud - Estados \\
& Dsicológicos - Respuesta Cognitiva \\
D4-F2. Síntomas subjetivos y alteraciones de la salud - Estados & psicológicos - Respuesta Conductual \\
D4. Desgaste Psíquico & Dsicos - Respuesta Fisiológica \\
\hline
\end{tabular}

Nota. Las dimensiones, factores globales, D1. Contexto de Trabajo; D2. Contenido de Trabajo y D3. Factores Individuales, así como los factores psicosociales específicos que las integran son las variables independientes, mientras que la D4. Desgaste Psíquico y los factores que agrupa son las variables dependientes. Elaboración propia a partir de la batería de CTCPS-MAC (Carrión, 2014).

Desgaste Psíquico es la variable dependiente. De igual manera, los factores psicosociales específicos de las primeras tres dimensiones son variables independientes, los de la cuarta dimensión son variables dependientes (véase Tabla 1).

\section{Procedimiento}

Se presentó el proyecto a la directiva de la institución, una vez aceptado, se comunicó por diversos medios la realización del estudio, así como el mecanismo, los horarios y espacios para participar. A quienes acudieron y firmaron el consentimiento informado, se les aplicó la batería CTCPS-MAC por evaluadores entrenados, siguiendo un guion introductorio y acorde a las indicaciones de esta, tales como: asegurar condiciones de confort, que el cuestionario fuera autoadministrado para quedar a disposición en caso de dudas sobre las preguntas, o bien, por entrevista a quien lo solicitara y verificar el llenado completo del cuestionario.

\section{Estrategia de análisis}

Se analizaron los datos en IBM SPSS Statistics 21 luego de ser capturados y calificados y se obtuvo primero los datos descriptivos del perfil sociodemográfico de los participantes y de 
las dimensiones y factores psicosociales percibidos. Para cubrir los objetivos y responder a las preguntas de investigación planteadas, primero se identificaron los factores psicosociales favorables y los desfavorables, después se evaluó la asociación con el coeficiente de Pearson de cada una de las dimensiones D1. Contexto de Trabajo, D2. Contenido de Trabajo y D3. Factores Individuales con la dimensión D4. Desgaste Psíquico. Luego, se evaluó la influencia mediante una regresión lineal múltiple. En ambos análisis se calculó el tamaño del efecto con el software G*Power 3.1, así como la proporción de trabajadores que perciben las dimensiones desfavorables con Desgaste Psíquico mediante el análisis de Odds Ratio.

\section{Resultados}

Se identificaron las dimensiones o factores psicosociales globales y los específicos, percibidos por el personal como favorables y desfavorables, en la Tabla 2 se observa que los participantes mostraron mayor predominio de niveles de muy bueno y bueno para cada una de las cuatro dimensiones. La dimensión D3. Factores Individuales fue la más favorable con $97.6 \%$ de muy bueno y bueno, seguida de la D1. Contexto de Trabajo con $89.1 \%$, la de menor percepción como favorable fue la D2. Contenido de Trabajo con $81.9 \%$. Lo anterior, pudo contribuir para reducir la probabilidad de sufrir Desgaste Psíquico, dimensión percibida por el 93.8\% muy bueno y bueno. Sin embargo, existe un porcentaje que percibió una o más de las cuatro dimensiones como desfavorable cuya respuesta fue: normal, regular y nociva, por lo cual es necesario tomar medidas.

Por su parte, los factores psicosociales específicos que componen la batería fueron percibidos por los trabajadores como muy bueno y bueno en general (véase Tabla 3). El factor más favorable fue el D1-F4. Relaciones interpersonales en el trabajo con el 100\% de la percepción favorable cuyas respuestas fueron muy bueno y bueno; continuado por el D4-F2. Respuesta Conductual con 93.5\% seguido por el D3-F3. Características Personales con 93.1\% y el D3-F2. Características de la Organización con 92.8\%, cierra la cuenta con percepción favorable arriba de 90\%, el D1-F2 Papel o Rol en la Organización con el $91.5 \%$.Sin embargo, al analizar los datos por trabajador, se encontró que el $66 \%$ de ellos percibieron por lo menos un factor psicosocial específico desfavorable.

Los factores psicosociales con percepción más desfavorable, acumulando el nivel normal, regular y nocivo, fueron tres de la dimensión D2. Contenido de Trabajo, específicamente el D2-F4. Concepción de las Tareas del Puesto de Trabajo con 42.5\%; el D2-F1. Carga y Ritmo de Trabajo con 28.7\% y el D2-F2. Ambientes Laborales con 26.7\%; y uno de la dimensión D1. Contexto de Trabajo, el D1-F1. Cultura de la Organización y Gestión con 30.4\%. Lo anterior mostró la necesidad de medidas de mejora, acciones reparadoras e inmediatas para evitar el daño.

Tabla 2

Frecuencia y porcentaje de las dimensiones de la batería Condiciones de Trabajo de Carácter Psicosocial

\begin{tabular}{lccccccccccc}
\hline Niveles & \multicolumn{1}{c}{ Muy bueno } & \multicolumn{3}{c}{ Bueno } & \multicolumn{2}{c}{ Normal } & \multicolumn{2}{c}{ Regular } & \multicolumn{2}{c}{ Nocivo } \\
\cline { 2 - 12 } Dimensión & $f r$ & $\%$ & $f r$ & $\%$ & $f r$ & $\%$ & $f r$ & $\%$ & fr & $\%$ \\
\hline D1. Contexto de trabajo & 112 & 38.4 & 148 & 50.7 & 3 & 1.0 & 28 & 9.6 & 1 & 0.3 \\
D2. Contenido de trabajo & 72 & 24.7 & 167 & 57.2 & 6 & 2.0 & 47 & 16.1 & 0 & 0 \\
D3. Factores individuales & 164 & 56.2 & 121 & 41.4 & 0 & 0 & 5 & 1.7 & 2 & 0.7 \\
D4. Desgaste psíquico & 136 & 46.6 & 138 & 47.2 & 2 & 0.7 & 14 & 4.8 & 2 & 0.7 \\
\hline Nota. Adaptado de batería Condiciones de Trabajo de Carácter Psicosocial (Carrión et al., 2014).
\end{tabular}

Nota. Adaptado de batería Condiciones de Trabajo de Carácter Psicosocial (Carrión et al., 2014). 
Tabla 3

Frecuencia y porcentaje de los factores de la Batería Condiciones de Trabajo de Carácter Psicosocial

\begin{tabular}{|c|c|c|c|c|c|c|c|c|c|c|}
\hline \multirow[b]{2}{*}{ Factor } & \multicolumn{2}{|c|}{ Muy bueno } & \multicolumn{2}{|c|}{ Bueno } & \multicolumn{2}{|c|}{ Normal } & \multicolumn{2}{|c|}{ Regular } & \multicolumn{2}{|c|}{ Nocivo } \\
\hline & $f r$ & $\%$ & $f r$ & $\%$ & $f r$ & $\%$ & $f r$ & $\%$ & $f r$ & $\%$ \\
\hline $\begin{array}{l}\text { D1-F1. Cultura de la } \\
\text { Organización y Gestión }\end{array}$ & 76 & 26.0 & 127 & 43.6 & 17 & 5.8 & 64 & 21.9 & 8 & 2.7 \\
\hline $\begin{array}{l}\text { D1-F2. Papel o Rol en la } \\
\text { Organización }\end{array}$ & 152 & 52.1 & 115 & 39.4 & 7 & 2.3 & 16 & 5.5 & 2 & 0.7 \\
\hline $\begin{array}{l}\text { D1-F3. Interrelación } \\
\text { Trabajo con Problemas } \\
\text { Familiares o Sociales }\end{array}$ & 116 & 39.7 & 118 & 40.4 & 25 & 8.6 & 28 & 9.6 & 5 & 1.7 \\
\hline $\begin{array}{l}\text { D1-F4. Relaciones } \\
\text { Interpersonales }\end{array}$ & 257 & 88.0 & 35 & 12.0 & 0 & 0 & 0 & 0 & 0 & 0 \\
\hline $\begin{array}{l}\text { D2-F1. Carga y Ritmo de } \\
\text { Trabajo }\end{array}$ & 78 & 26.8 & 130 & 44.5 & 10 & 3.4 & 61 & 20.9 & 13 & 4.4 \\
\hline $\begin{array}{l}\text { D2-F2. Ambientes } \\
\text { Laborales }\end{array}$ & 82 & 28.4 & 132 & 45.2 & 15 & 5.1 & 44 & 14.8 & 19 & 6.5 \\
\hline $\begin{array}{l}\text { D2-F3. Equipos y Agentes } \\
\text { Físicos }\end{array}$ & 122 & 41.8 & 120 & 41.1 & 16 & 5.5 & 31 & 10.6 & 3 & 1.0 \\
\hline $\begin{array}{l}\text { D2-F4. Concepción de } \\
\text { las Tareas del Puesto de } \\
\text { Trabajo }\end{array}$ & 87 & 29.8 & 81 & 27.7 & 61 & 20.9 & 51 & 17.5 & 12 & 4.1 \\
\hline $\begin{array}{l}\text { D3-F1. Amortiguadores } \\
\text { del Riesgo Psíquico }\end{array}$ & 155 & 53.1 & 106 & 36.3 & 4 & 1.4 & 24 & 8.2 & 3 & 1.0 \\
\hline $\begin{array}{l}\text { D3-F2. Características de } \\
\text { la Organización }\end{array}$ & 170 & 58.2 & 101 & 34.6 & 9 & 3.1 & 9 & 3.1 & 3 & 1.0 \\
\hline $\begin{array}{l}\text { D3-F3. Características } \\
\text { Personales }\end{array}$ & 144 & 49.4 & 128 & 43.8 & 10 & 3.4 & 7 & 2.4 & 3 & 1.0 \\
\hline $\begin{array}{l}\text { D4-F1. Respuesta } \\
\text { Cognitiva-Emocional }\end{array}$ & 99 & 33.9 & 131 & 44.9 & 11 & 3.8 & 39 & 13.3 & 12 & 4.1 \\
\hline $\begin{array}{l}\text { D4-F2. Respuesta } \\
\text { Conductual }\end{array}$ & 179 & 61.3 & 94 & 32.2 & 6 & 2.1 & 8 & 2.7 & 5 & 1.7 \\
\hline $\begin{array}{l}\text { D4-F3. Respuesta } \\
\text { Fisiológica }\end{array}$ & 155 & 53.1 & 95 & 32.5 & 4 & 1.4 & 33 & 11.3 & 5 & 1.7 \\
\hline
\end{tabular}

Nota. Adaptado de batería Condiciones de Trabajo de Carácter Psicosocial (Carrión et al., 2014).

Se obtuvo la correlación de Pearson de las dimensiones D1. Contexto de Trabajo, D2. Contenido de Trabajo y D3. Factores Individuales con D4. Desgaste Psíquico. Los resultados indicaron una correlación media, positiva y estadísticamente significativa (Coolican, 2005), por lo cual se demuestra la asociación entre las condiciones laborales del contexto y contenido de trabajo y los factores individuales con las respuestas cognitiva-emocional, conductual y fisiológica. La variable con mayor fuerza en la correlación fue D3. Factores Individuales (véase Tabla 4).

Se efectuó un análisis de asociación Odds Ratio o Razón de Momios entre cada dimensión psicosocial y el Desgaste Psíquico, el cual permite comparar el grupo de trabajadores 
Tabla 4

Correlación de Pearson entre las dimensiones psicosociales de la Batería Condiciones de Trabajo de Carácter Psicosocial y el Desgaste Psíquico

\begin{tabular}{|c|c|c|c|c|c|}
\hline & & $\begin{array}{c}\text { D1. Contexto } \\
\text { de Trabajo }\end{array}$ & $\begin{array}{l}\text { D2. Contenido } \\
\text { de Trabajo }\end{array}$ & $\begin{array}{l}\text { D3. Factores } \\
\text { Individuales }\end{array}$ & $\begin{array}{l}\text { D4. Desgaste } \\
\text { Psíquico }\end{array}$ \\
\hline \multirow[t]{2}{*}{$\begin{array}{l}\text { D1. Contexto } \\
\text { de Trabajo }\end{array}$} & $\begin{array}{l}\text { Coeficiente de } \\
\text { correlación }\end{array}$ & - & $.46^{\star}$ & $.62^{*}$ & $.47^{\star}$ \\
\hline & $\begin{array}{l}\text { Tamaño del } \\
\text { efecto }\end{array}$ & - & .68 & .79 & .68 \\
\hline \multirow[t]{2}{*}{$\begin{array}{l}\text { D2. Contenido } \\
\text { de Trabajo }\end{array}$} & $\begin{array}{l}\text { Coeficiente de } \\
\text { correlación }\end{array}$ & & - & $.41^{\star}$ & $.41^{*}$ \\
\hline & $\begin{array}{l}\text { Tamaño del } \\
\text { efecto }\end{array}$ & & - & .64 & .64 \\
\hline \multirow[t]{2}{*}{$\begin{array}{l}\text { D3. Factores } \\
\text { Individuales }\end{array}$} & $\begin{array}{l}\text { Coeficiente de } \\
\text { correlación }\end{array}$ & & & - & $.52^{*}$ \\
\hline & $\begin{array}{l}\text { Tamaño del } \\
\text { efecto }\end{array}$ & & & - & .72 \\
\hline
\end{tabular}

Nota. Adaptado de la batería Condiciones de Trabajo de Carácter Psicosocial (Carrión et al., 2014). ${ }^{*} p<.01$

con percepción desfavorable de las dimensiones psicosociales y percepción desfavorable de Desgaste Psíquico con respecto a los que, teniendo percepción desfavorable de las dimensiones psicosociales, tuvieron percepción favorable de Desgaste Psíquico. Se encontró que en proporción, los primeros fueron 16.45, 13.77 y 30.33 veces más que los segundos. Al ser una razón de momios, se categorizaron las respuestas de percepción muy bueno y bueno como favorable y las de normal, regular y nocivo como desfavorable. Los resultados fueron positivos y estadísticamente significativos $(p<.01)$ y se muestran para cada dimensión en las Tablas 5, 6 y 7.

Se corrió una regresión lineal múltiple con método Stepwise por orden de acomodo de las dimensiones de la batería, la dimensión psicosocial con mayor influencia en el Desgaste Psíquico de la población de estudio fue D3. Factores Individuales seguida de D2. Contenido de Trabajo, y D1. Contexto de Trabajo con Coeficiente Beta Estandarizado de .33, .20 y .17 respectivamente, todas con significancia estadística $(p<.01)$ (véase Tabla 8 ).

El modelo en su conjunto explica el 33\% del Desgaste Psíquico de acuerdo con el coeficiente de determinación ( $\left.\Delta R^{2}=.33\right)$. El tamaño del efecto para la regresión de 49 es grande ya que es de 35 o mayor (Domínguez-Lara, 2018). Los coeficientes de colinealidad de Tolerancia fueron superiores a .20 y los VIF (Variance inflation factor) inferiores a 10 como lo sugiere la literatura (Cea D'Ancona, 2004), lo anterior indica que no existe correlaciones elevadas entre las dimensiones del modelo. Por último, el indicador Drubin Watson se ubicó dentro de 1.5 y 2.5 lo que indica que existe homocedasticidad (Cea D’Ancona, 2004). 
Tabla 5

Análisis de Odds Ratio de Contexto de Trabajo versus Desgaste Psíquico

\begin{tabular}{lcccccc}
\hline & & \multicolumn{2}{c}{ Desgaste psíquico } & ORc & \multirow{2}{*}{ X2 } & $p$ \\
\cline { 3 - 5 } Percepción & & Sí & No & (IC 95\%) & & \\
\hline Contexto de Trabajo desfavorable & Sí & 9 (a) & 20 (b) & 16.45 & 40.59 & .001 \\
& No & 7 (c) & 256 (d) & $(5.54-48.82)$ & & \\
& & & & & &
\end{tabular}

Nota. $N=292$. ORc $=$ Odds Ratio, IC = Intervalo de Confianza, $\chi^{2}=$ Chi cuadrada.

Tabla 6

Análisis de Odds Ratio de Contexto de Trabajo versus Desgaste Psíquico

\begin{tabular}{lcccccc}
\hline & & \multicolumn{2}{c}{ Desgaste psíquico } & ORc & \multirow{2}{*}{ X2 } & $p$ \\
\cline { 2 - 5 } Percepción & & Sí & No & (IC 95\%) & & \\
\hline Contexto de Trabajo desfavorable & Sí & $11(\mathrm{a})$ & $38(\mathrm{~b})$ & 13.77 & 32.73 & .001 \\
& No & 5 (c) & 238 (d) & $(4.53-41.86)$ & & \\
\hline
\end{tabular}

Nota. $N=292$. ORc = Odds Ratio, IC = Intervalo de Confianza, $\chi 2=$ chi cuadrada.

Tabla 7

Análisis de Odds Ratio de Contexto de Trabajo versus Desgaste Psíquico

\begin{tabular}{|c|c|c|c|c|c|c|}
\hline \multirow[b]{2}{*}{ Percepción } & & \multicolumn{2}{|c|}{ Desgaste psíquico } & ORc & \multirow{2}{*}{$x^{2}$} & \multirow{2}{*}{$n$} \\
\hline & & Sí & No & (IC 95\%) & & \\
\hline \multirow[t]{2}{*}{ Contexto de trabajo desfavorable } & Sí & $4(\mathrm{a})$ & $12(b)$ & \multirow{2}{*}{$\begin{array}{c}30.33 \\
(6.09-150.94)\end{array}$} & \multirow[t]{2}{*}{36.96} & \multirow[t]{2}{*}{.001} \\
\hline & No & $3(c)$ & $273(d)$ & & & \\
\hline
\end{tabular}

Nota. $N=292$. ORc = Odds Ratio, IC = Intervalo de Confianza, $x 2=$ chi cuadrada.

Tabla 8

Análisis de regresión lineal múltiple de las dimensiones con el Desgaste Psíquico

\begin{tabular}{lcccccc}
\hline & \multicolumn{5}{c}{ Desgaste Psíquico (variable dependiente) } \\
\cline { 2 - 6 } & $B$ & $\Delta R^{2}$ & $t$ & $p$ & $F$ & $f 2$ \\
\hline Variables independientes & & .33 & 4.14 & .00 & 49.13 & .49 \\
D1. Contexto de Trabajo & .17 & & 2.68 & .01 & & \\
D2. Contenido de Trabajo & .20 & & 3.68 & .00 & \\
D3. Factores Individuales & .33 & & 5.32 & .00 & & \\
\hline
\end{tabular}

Nota. $N=292$. $B=$ Coeficiente Beta Estandarizado, $R^{2}=$ Coeficiente de determinación, $t=$ prueba t de Student, $F=$ prueba $F$ de Fisher, $f 2$ = Tamaño del efecto.

Actualidades en Psicología, 35(130), 2021 115-130 


\section{Discusión}

Los factores psicosociales empiezan a causar mayor interés de las organizaciones por su influencia en la salud de los trabajadores y en su desempeño (Moreno y Báez, 2010), razón por la cual se conceptualizaron y establecieron lineamientos internacionales (Agencia Europea para la Seguridad y la Salud en el Trabajo, 2016; Comité Mixto de la OIT/OMS de Medicina del Trabajo, 1984; OMS, 2006). Las legislaciones de seguridad y salud en el trabajo de muchos países (INSHT, 2014; Norma Oficial Mexicana NOM-035-STPS-2018, 2018), así como investigadores e instituciones (Burr et al., 2019; Carrión, 2014; Martín et al., 1995; ISTAS, 2016) los retoman con este y otros términos relacionados, lo que es importante considerar al momento de su estudio.

El fundamento de la explicación en la relación de las condiciones de trabajo con la salud incluye los enfoques técnico, fisiológico y psicológico (Karasek, 1979; Lazarus, 1974; Selye, 1946), este último predomina como antecedente teórico de los lineamientos de la OIT (Cox et al., 2005) y de los marcos jurídicos nacionales, que buscan garantizar los derechos humanos a la salud y el trabajo, en línea con el tratamiento que se da a los otros factores de riesgo laboral en materia de seguridad y salud en el trabajo.

De acuerdo con el propósito de este estudio, se encontró que los factores psicosociales percibidos por los participantes fueron en su mayoría favorables tanto para las dimensiones o factores psicosociales globales como para los específicos que las integran, con respuestas de muy bueno y bueno (Andersen et al., 2020; Gutiérrez, 2011; OMS, 2017).

Los factores más favorables fueron de las dimensiones Factores Individuales y Contexto de Trabajo, así como en el factor específico de Respuesta Conductual, por lo cual las buenas prácticas de la institución educativa deben conservarse y enriquecerse como medidas preventivas y promoción de un entorno organizacional favorable (Chiavenato, 2011; Norma Oficial Mexicana NOM-035-STPS-2018, 2018; Patlán, 2016; Tamayo et al., 2014) con perspectiva de gestión integral (Anaya, 2017; Carrión et al., 2008; ISO, 2010; LaMontagne et al., 2014; OMS, 2010; Salanova et al., 2013) para potenciar sus beneficios en la salud, el desempeño del personal y de la institución.

Los factores psicosociales específicos con percepción más desfavorable fueron de la dimensión Contenido de Trabajo, Concepción de las Tareas del Puesto de Trabajo, Carga y Ritmo de Trabajo y el de Cultura de la Organización y Gestión del Contexto de Trabajo, lo que se relacionó con Desgaste Psíquico en la Respuesta Cognitiva-Emocional para un quinto de los trabajadores, además el 66\% percibieron por lo menos uno de los 14 factores psicosociales específicos desfavorable.

Lo anterior pone de manifiesto la importancia del estudio de los puestos de trabajo tal como lo indica el Reglamento Federal de Seguridad y Salud en el Trabajo (2014) y la Norma Oficial Mexicana NOM-035-STPS-2018 (2018), tanto desde las condiciones del ambiente físico, como de la organización del trabajo, para su adecuación o rediseño, ya que influyen en el bienestar y el desempeño percibidos. También, pueden relacionarse con quejas, deseo de abandono de la organización con afectación al trabajador, además, los costos administrativos y riesgo de impactar en las metas organizacionales (Cacivio, 2017; Hammer et al., 2015; Ramalho \& Costa, 2017).

El hallazgo anterior coincide con lo encontrado por Saldaña et al. (2014) en un estudio realizado en una institución pública, en ambos se plantean acciones dirigidas a mejorar, 
concretamente en la organización del trabajo, la definición de responsabilidades, la comunicación y opciones de participación.

En este sentido, la satisfacción laboral se asocia con la calidad de vida en el trabajo, por lo cual, prestar atención al bienestar del personal y al entorno de trabajo favorable es una vía para ser una organización saludable, las cuales se caracterizan por ser económicamente estables, con trabajadores altamente motivados y con clientes satisfechos (Salanova et al., 2013; Salanova et al., 2021).

Las dimensiones Contexto de Trabajo, Contenido de Trabajo y Factores Individuales correlacionaron con el Desgaste Psíquico, de manera estadísticamente significativa, en un nivel medio (Coolican, 2005), con tamaño de efecto grande (Domínguez-Lara, 2018) lo cual indica que dichas dimensiones son presumiblemente predictoras para padecer Desgaste Psíquico (Carrión, 2014; Xiao et al., 2020).

El análisis de Odds Ratio confirmó una asociación, en concordancia con el enfoque teórico psicológico de la relación de condiciones de trabajo y salud de los empleados, ya sea favorable o desfavorable. El hallazgo de correlación del estudio, similar a lo encontrado por Carrión et al. (2014) y la asociación fue en el mismo sentido.

Es explicable que al identificar en un estudio factores psicosociales desfavorables dada su correlación con la salud, la normativa establezca la obligación de actuar con medidas preventivas a nivel primario en toda la organización, a nivel secundario en grupos con exposición y a nivel terciario dar atención a los trabajadores con riesgo psicosocial con medidas correctoras inmediatas (Norma Oficial Mexicana NOM-035-STPS-2018, 2018; Patlán, 2016; Tomascheck et al., 2017; Vestly et al., 2017).

De acuerdo con el análisis de regresión, se infiere que Contexto de Trabajo, Contenido de Trabajo y Factores Individuales afectan a la variable dependiente, Desgaste Psíquico, es decir, en la respuesta cognitiva-emocional, conductual y fisiológica de los trabajadores.

En la población de estudio la dimensión psicosocial que más influyó fue Factores Individuales (Coeficiente Beta Estandarizado .33) y fue favorable (97.6\%). El modelo en su conjunto explica el 33\% del Desgaste Psíquico de acuerdo con el coeficiente de determinación $(\triangle R 2=.33)$. Se sugiere hacer otros planteamientos al modelo ya que no aclara cabalmente el Desgaste Psíquico. Lo explicado por el modelo en su totalidad es mayor al 12\% encontrado en el modelo de Carrión et al. (2014) que contempló variables sociodemográficas y laborales de los participantes.

Los instrumentos de evaluación de factores psicosociales deben tener evidencia de validez y ser confiables para que permitan el diagnóstico psicosocial de la organización o áreas parciales de la misma, desde la percepción individual de cada trabajador. Es necesario atender minuciosamente las indicaciones y condiciones de aplicación pues son bajo las cuales probaron ser válidos y confiables.

Los métodos que proponen los organismos internacionales y los marcos jurídicos nacionales, en general son multidimensionales y expresan la necesidad de realizar cuando sea necesario, estudios específicos de cada factor prioritario para el bienestar y mejor desempeño del trabajador, así como para los resultados de la organización a fin de un mejor análisis que permita establecer medidas pertinentes y eficaces, con la participación de los trabajadores (Martín et al., 1995; ISTAS, 2015; Carrión et al., 2014; Norma Oficial Mexicana NOM-035-STPS-2018, 2018). 
Para mejorar la explicación del diagnóstico psicosocial cuantitativo, resulta necesario utilizar metodología cualitativa con las expresiones y datos emergidos de los propios trabajadores de todos los niveles jerárquicos, categorizar la información y analizarla como lo señalan Abildgaard et al. (2016). De igual manera, son importantes las estrategias participativas para la identificación de los factores de riesgo, la propuesta de medidas de mejora y la ejecución de estas, en especial los grupos expuestos (Martín et al., 1995; ISTAS, 2015; Carrión et al., 2014; Norma Oficial Mexicana NOM-035-STPS-2018, 2018).

Los factores psicosociales deberán evaluarse periódicamente. Al respecto, la NOM-035STPS-2018 señala dos años para establecer medidas que permitan mantener los factores favorables y las acciones pertinentes para los desfavorables. Como lo señala Ansoleaga (2015) es indispensable el monitoreo y una posterior evaluación para conocer los avances de la gestión.

Para un entorno organizacional saludable que reduzca al mínimo los factores de riesgo psicosocial y potencie los favorables como en la institución del estudio, se propone interiorizar una cultura de gestión integral y de trabajo colaborativo, en la cual los administradores y el personal de las organizaciones consideren en un mismo nivel y en sinergia, la salud y la seguridad del personal, el cuidado del entorno natural y social, la calidad y la productividad, lo cual ya es explícito en el marco jurídico mexicano en esas materias (Ley Federal del Trabajo, 2012; Reglamento Federal de Seguridad y Salud en el Trabajo, 2014; Norma Oficial Mexicana NOM-035-STPS-2018, 2018), dirigiendo sus análisis, decisiones y acciones tanto individuales como colectivas, en consideración de todas las partes interesadas dada su declarada responsabilidad social universitaria.

Los requerimientos para la acción desde la gestión integral organizacional van dirigidos hacia un cambio sustentable, tanto desde el liderazgo como en la autorresponsabilidad del personal sobre su propio bienestar. Por ello, la comunicación, la información, la formación y la participación son determinantes para mantener o mejorar la salud y el desempeño de los trabajadores que contribuyan a las metas de la organización y a la sociedad en su conjunto.

Una limitante del estudio es que al ser voluntaria la participación, quienes no aceptaron probablemente tienen una percepción diferente. En investigaciones futuras para mejorar las explicaciones, es conveniente: cubrir a toda la población, por los casos de alto riesgo, o mínimo una muestra probabilística, realizar estudios con enfoque cualitativo, con recolección de indicadores objetivos para cruce de información y de diseño longitudinal.

\section{Referencias}

Abildgaard, J., Saksvik, P., \& Nielsen, K. (2016). How to measure the intervention process? An assessment of qualitative and quantitative approaches to data collection in the process evaluation of organizational interventions. Frontiers in Psychology, 7(1380), 1-10. https://doi.org/10.3389/fpsyg.2016.01380

Agencia Europea para la Seguridad y la Salud en el Trabajo. (2016). Los riesgos psicosociales y el estrés en el trabajo, nota descriptiva. https://osha.europa.eu/es/themes/ psychosocial-risks-and-stress

Anaya-Velasco, A. (2017). Modelo de Salud y Seguridad en el Trabajo con Gestión Integral para la Sustentabilidad en las Organizaciones (SSeTGIS). Ciencia y Trabajo, 19(59), 95-104. http://dx.doi.org/10.4067/S0718-24492017000200095 
Andersen, L. L., Villadsen, E., \& Clausen, T. (2020). Influence of physical and psychosocial working conditions for the risk of disability pension among healthy female eldercare workers: prospective cohort. Scandinavian Journal of Public Health, 48(4), 460-467. https://doi.org/10.1177/1403494819831821

Ansoleaga, E. (2015). Indicadores de salud mental asociados a riesgo psicosocial laboral en un hospital público. Revista Médica de Chile, 143(1), 47-55. http://dx.doi. org/10.4067/S0034-98872015000100006

Berthelsen, H., Westerlund, H., Bergström, G., \& Burr, H. (2020). Validation of the Copenhagen psychosocial questionnaire version III and establishment of benchmarks for psychosocial risk management in Sweden. International Journal of Environmental Research and Public Health, 17(9). https://doi.org/10.3390/ ijerph17093179

Burr, H., Berthelsen, H., Moncada, S., Nübling, M., Dupret, E., Demiral, Y., Oudyk, J., Kristensen, T. S., Llorens, C., Navarro, A., Lincke, H. J., Bocéréan, C., Sahan, C., Smith, P., \& Pohrt, A. (2019). The third version of the Copenhagen psychosocial questionnaire. Safety and Health at Work, 10(4), 482-503. https://doi.org/10.1016/j. shaw.2019.10.002

Cacivio, R. (2017). Hablemos de nuestro trabajo. Riesgos psicosociales en entornos de trabajo de extensionistas agropecuarios de la Argentina. Pesquisa Empírica, 13(1), 39-55. https://doi.org/10.4000/laboreal.1886

Carrión-García, M. (2014). Batería para el estudio de las condiciones de trabajo de carácter psicosocial. Editorial Académica Española.

Carrión-García, M., Gutiérrez, A., \& López, F. (2014). Condiciones de trabajo psicosociales y desgaste psíquico en trabajadores de diversos sectores empresariales CentroOriente, Suroccidente y región Caribe en Colombia. Revista Científica Salud Uninorte, 30(3), 311-322. http://dx.doi.org/10.14482/sun.30.1.4309

Carrión-García, M., López, F., \& Tous, J. (2008). El modelo AEPA protocolo para la intervención psicosocial. Más allá de la evaluación psicosocial. Seguridad y Salud en el Trabajo, 45, 22-30. https://app.mapfre.com/documentacion/pt/catalogo_ imagenes/grupo.do?path $=1054615$

Cea D’Ancona, M. A. (2004). Análisis Multivariable. Teoría y práctica en la investigación social. Síntesis.

Chiavenato, I. (2011). Administración de recursos humanos, el capital humano en las organizaciones. Mc Graw Hill.

Coolican, H. (2005). Métodos de investigación y estadística en psicología. (3era Ed). Manual Moderno.

Cox, T., Griffiths, A., \& Rial-González, E. (2005). Investigación sobre el estrés relacionado con el trabajo. https://osha.europa.eu/es/publications/report-research-work-related-stress

Comité Mixto de la OIT/OMS de Medicina del Trabajo. (1984). Factores psicosociales en el trabajo: naturaleza, incidencia y prevención. Oficina Internacional del Trabajo. http:// www.factorespsicosociales.com/wp-content/uploads/2019/02/FPS-OIT-OMS.pdf

Domínguez-Lara, S. (2018). Magnitud del efecto, una guía rápida. Educación Médica, 19(4), 251-254. http://dx.doi.org/10.1016/j.edumed.2017.07.002

Actualidades en Psicología, 35(130), 2021 115-130 
Gutiérrez, A. (2011). Guía técnica para el análisis de exposición a factores de riesgo ocupacional para el proceso de evaluación en la calificación de origen de la enfermedad profesional. Ministerio de la protección social \& Imprenta Nacional de Colombia. https://comunicandosalud.com/wp-content/uploads/2019/06/ guia_exposicion_factores_riesgo_ocupacional.pdf

Hammer, L., Truxillo, D., Bodner, T., Rineer, J., Pytlovany, A., \& Richman, A. (2015). Effects of a workplace intervention targeting psychosocial risk factors on safety and health outcomes. BioMed Research International, (836967), 1-12 http://dx.doi. org/10.1155/2015/836967

Instituto Nacional de Seguridad e Higiene en el Trabajo. (2014). Método de evaluación F-PSICO.

Instituto Sindical del Trabajo, Ambiente y Salud. (2015). Riesgos Psicosociales. https://istas. net/salud-laboral/peligros-y-riesgos-laborales/riesgos-psicosociales

Instituto Sindical del Trabajo, Ambiente y Salud. (2016). CoPsoQ-istas21. https://copsoq. istas21.net/index.asp?ra_id $=46$

Karasek, R. A. J. (1979). Job Demands, Job Decision Latitude, and Mental Strain: Implications for Job Redesign. Administrative Science Quarterly, 24(2), 285-308. http://www. jstor.org/stable/2392498

LaMontagne, A., Martin, A., Page, K., Reavley, N., Noblet, a., Milner, A., Keegel, T., \& Smith, P. (2014). Workplace mental health: developing an integrated intervention approach. BMC Psychiatry, 14, 131. https://doi.org/10.1186/1471-244X-14-131

Lazarus, R. S. (1974). Psychological stress and coping in adaptation and illness. The International Journal of Psychiatry in Medicine, 5(4), 321-333. https://doi. org/10.2190/t43t-84p3-qdur-7rtp

Ley Federal del Trabajo. Diario Oficial de la Federación. (2012). http://dof.gob.mx/nota_ detalle.php?codigo $=5280815 \&$ fecha $=30 / 11 / 2012$

Martín, F. D., \& Pérez, J. B. (1995). Factores psicosociales: metodología de evaluación. Instituto Nacional de Seguridad e Higiene en el Trabajo. http://www.insht.es/InshtWeb/ Contenidos/Documentacion/FichasTecnicas/NTP/Ficheros/401a500/ntp_443.pdf

Moreno, B., \& Báez, C. (2010). Factores y riesgos psicosociales, formas, consecuencias, medidas y buenas prácticas. Instituto Nacional de Seguridad e Higiene en el Trabajo. https:// www.insst.es/documentacion/catalogo-de-publicaciones/factores-y-riesgospsicosociales-formas-consecuencias-medidas-y-buenas-practicas

Norma Oficial Mexicana NOM-035-STPS-2018, Factores de riesgo psicosocial en el trabajoIdentificación, análisis y prevención. (2018). https://www.dof.gob.mx/nota_detalle. php? codigo $=5541828 \&$ fecha $=23 / 10 / 2018$

Organización Internacional del Trabajo. (2021). Anticiparse a las crisis, prepararse y responder. Invertir hoy en sistemas resilientes de SST. https://www.ilo.org/wcmsp5/groups/ public/---ed_dialogue/---lab_admin/documents/publication/wcms_780968.pdf

Organización Internacional de Normalización. (2010). Norma Internacional: guía de responsabilidad social.

Organización Mundial de la Salud. (2006). El informe sobre la salud en el mundo 2006: Colaboremos por la salud. http://www.who.int/whr/2006/chapter1/es/ 
Organización Mundial de la Salud. (2010). Entornos laborales saludables: fundamentos y modelo de la OMS. Contextualización, prácticas, y literatura de apoyo. https:// www.who.int/occupational_health/evelyn_hwp_spanish.pdf

Organización Mundial de Salud. (2017). Factores de riesgo. http://www.who.int/topics/ risk_factors/es/

Patlán, J. (2016). Calidad de vida en el Trabajo. Manual Moderno.

Ramalho, J., \& Costa, L. (2017). Os fatores psicossociais de risco na atividade de técnicos superiores de segurança no trabalho. Laboreal, 13(2), 39-49.

Reglamento Federal de Seguridad y Salud en el Trabajo. Disposiciones Generales, Competencias y Sujetos Obligados. Diario Oficial de la Federación. (2014). http:// www.diputados.gob.mx/LeyesBiblio/regla/n152.pdf

Salanova, M., Acosta-Antognoni, H., Llorens, S., \& Le Blanc, P. (2021). We trust you! a multilevel-multireferent model based on organizational trust to explain performance. International Journal of Environmental Research and Public Health, 18(8). https://doi.org/10.3390/ijerph18084241

Salanova, M., Llorens, S., Acosta, H., \& Torrente, P. (2013). Positive interventions in positive organizations. Terapia Psicológica, 31(1), 101-113. http://dx.doi.org/10.4067/S071848082013000100010

Saldaña, C., Ramírez, E., Anaya-Velasco, A., \& Baeza, M. (2014). Evaluación de los riesgos psicosociales con el instrumento CTCPS-MAC batería para el estudio de las condiciones de trabajo de carácter psicosocial- en el Departamento de Obras Públicas de un ayuntamiento del sur de Jalisco (México). Revista Científica Salud Uninorte, 30(3), 293-301. https://www.redalyc.org/articulo.oa?id=81737153004

Selye, H. (1946). The general adaptation syndrome and the diseases of adaptation. The Journal of Clinical Endocrinology \& Metabolism, 6(2), 117-230. https://doi. org/10.1210/jcem-6-2-117

Tamayo, Y., Del Río, A., \& García, D. (2014). Modelo de gestión organizacional basado en el logro de objetivos. Suma de Negocios, 5(11), 70-77. https://doi.org/10.1016/ S2215-910X(14)70021-7

Tomascheck, A., Lütke, S., Melzer, M., Debitz, U., \& Buruck, G. (2017). Measuring workrelated psychosocial and physical risk factors using workplace observations: a validation study of the "Healthy Workplace Screening". Safety Science, 101,197208. https://doi.org/10.1016/j.ssci.2017.09.006

Vestly, L., Leka, S., \& Zwetsloot, G. (2017). Tailoring Psychosocial Risk Assessment in the Oil and Gas Industry by Exploring Specific and Common Psychosocial Risks. Safety and Health at Work, 9(1), 63-70. https://doi.org/10.1016/j.shaw.2017.05.001

Xiao, Y., Zhang, T., Gu, X., Lee, J., \& Wang, H. (2020). The Roles of Individual and Psychosocial Factors in Predicting Quality of Life Among Working Women in Shanghai. International Journal of Environmental Research and Public Health, 17(5). https://doi.org/10.3390/ijerph17051751 